\title{
molecules
}

ISSN 1420-3049

http://www.mdpi.org

\section{Aminoacids in the Synthesis of Heterocyclic Systems: The Synthesis of Triazinoquinazolinones, Triazepinoquinazolinones and Triazocinoquinazolinones of Potential Biological Interest}

\author{
A.M.Sh.El-Sharief ${ }^{1, a}$, Y.A.Ammar ${ }^{1, *}$, M.A.Zahran ${ }^{1}$, A.H.Ali ${ }^{1}$ and M.S.A.El-Gaby ${ }^{2, b}$ \\ ${ }^{1}$ Department of Chemistry, Faculty of Science, Al-Azhar University, Nasr-City 11884, Cairo, Egypt. \\ aE-mail: ahmed_shab@hotmail.com; Fax: 002(02)2629356 \\ ${ }^{2}$ Department of Chemistry, Faculty of Science, Al-Azhar University at Assiut, Assiut 71524 Egypt. \\ bE-mail: $\underline{\text { m_elgaby@ hotmail.com, Fax: +20 } 88325436}$
}

*Author to whom correspondence should be addressed; E-mail: yossry@yahoo.com

Received: 5 April 2000; in revised form 29 August 2000 / Accepted: 1 February 2001 / Published: 28 February 2001

\begin{abstract}
A number of novel triazinoquinazolinones (5b,c and 8), triazepinoquinazolinones $(\mathbf{5 a}, \mathbf{6 b}, \mathbf{7}$ and 9) and triazocinoquinazolinones (6a and 10) were obtained via nucleophilic interaction of 3-aminoquinazolinone derivatives 3 with different reagents.
\end{abstract}

Keywords: Triazinoquinazolinones, triazepinoquinazolinones, triazocinoquinazolinones, biological activity

\section{Introduction}

Varied biological activities have been attributed to quinazoline compounds, including analgesic, antiinflammatory, antipyretic [1-3], antimicrobial [4], anticonvulsant [5], fungicidal [6], antidepressant and other central nervous system affecting activities [7]. The use of aminoacids as starting materials for the design and synthesis of new quinazoline compounds with the aim of preparing potent biologically active compounds is a subject of recent interest [8-11]. Now we report a facile synthesis of several heterocyclic compounds containing six, seven and eight membered rings fused to a quinazoline moiety starting from amino acids. 


\section{Results and Discussion.}

\section{Synthesis and characterization}

The starting materials 1a-f were prepared via reaction of the appropriate sulfonyl chloride derivatives with amino acids in the presence of $10 \%$ sodium hydroxide solution, followed by acidification [12]. The amides $\mathbf{2 a - f}$ have been prepared by condensation of the corresponding 1a-f with methyl anthranilate in presence of $\mathrm{PCl}_{3}$. 3-Aminoquinazolinones 3a-f were obtained by refluxing compounds 2a-f with hydrazine hydrate in n-butanol (Scheme 1). Compounds 2a-f and 3af have been fully characterized by their analytical and spectral data. For example, the IR spectrum of compound 3e showed the presence of $\mathrm{NH}, \mathrm{NH}_{2}, \mathrm{CH}$-aliphatic, $\mathrm{C}=\mathrm{O}$ and $\mathrm{S}=\mathrm{O}$ functional groups. Also, the ${ }^{1} \mathrm{H}-\mathrm{NMR}$ spectrum of compound $3 \mathbf{e}$ exhibited signals in the range of $0.85-0.92 ; 1.88 ; 2.15-2.24$; 2.50-2.51; 5.51; 6.82-8.02; 10.6 ppm due to $2 \mathrm{CH}_{3}(\mathrm{~d}) ; \mathrm{CH}_{3}(\mathrm{~s}) ; \mathrm{CH}(\mathrm{m}) ; \mathrm{CH}(\mathrm{d}) ; \mathrm{NH}_{2}$ (s); $\mathrm{Ar}-\mathrm{H}$ 's (m) and $\mathrm{SO}_{2} \mathrm{NH}$ (s) respectively. The mass spectrum of compound 3c showed a molecular ion peak at $\mathrm{m} / \mathrm{z}$ $434(1.01 \%)$ which underwent fragmentation to give the well established fragment at $\mathrm{m} / \mathrm{z} 91(100 \%$; base peak; see Scheme 2).

\section{Scheme 1}
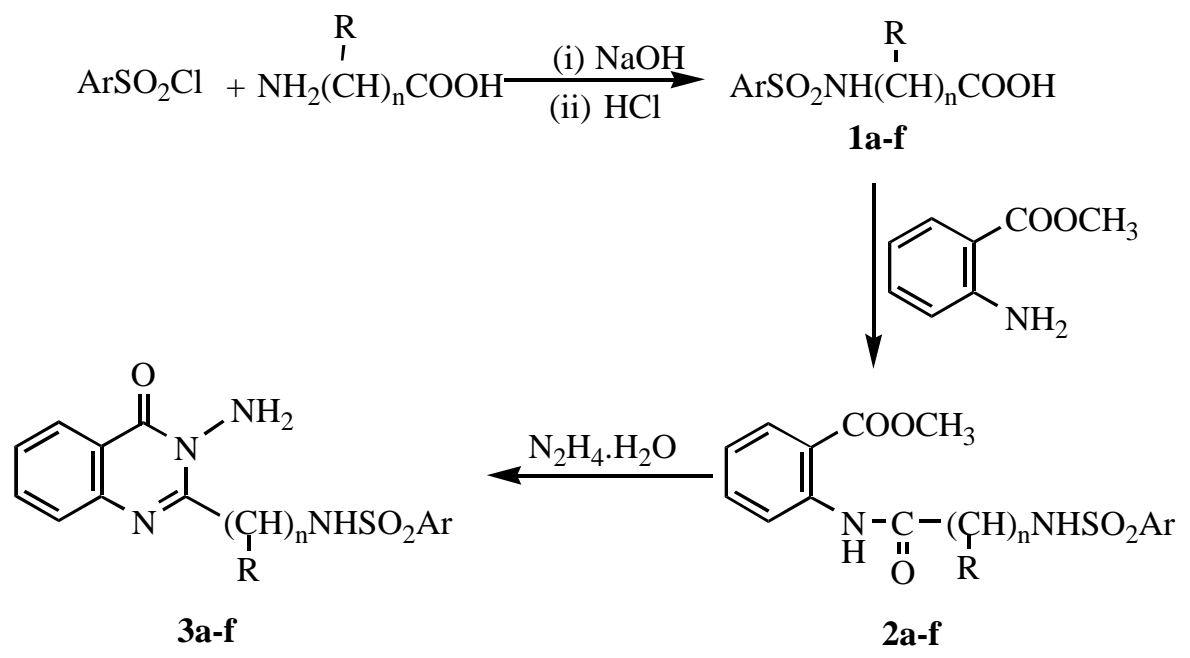

$\begin{array}{clll}\text { 1,2,3a } & \mathrm{R}=\mathrm{H} & \mathrm{Ar}=\mathrm{C}_{6} \mathrm{H}_{5} & \mathrm{n}=1 \\ \text { b } & \mathrm{R}=\mathrm{C}_{6} \mathrm{H}_{5} & \mathrm{Ar}=\mathrm{p}-\mathrm{CH}_{3}-\mathrm{C}_{6} \mathrm{H}_{4} & \mathrm{n}=1 \\ \text { c } & \mathrm{R}=\mathrm{CH}_{2} \mathrm{C}_{6} \mathrm{H}_{5} & \mathrm{Ar}=\mathrm{p}-\mathrm{CH}_{3}-\mathrm{C}_{6} \mathrm{H}_{4} & \mathrm{n}=1 \\ \text { d } & \mathrm{R}=\mathrm{H} & \mathrm{Ar}=\mathrm{p}-\mathrm{CH}_{3}-\mathrm{C}_{6} \mathrm{H}_{4} & \mathrm{n}=2 \\ \text { e } & \mathrm{R}=\mathrm{CH}\left(\mathrm{CH}_{3}\right)_{2} & \mathrm{Ar}=\mathrm{p}-\mathrm{CH}_{3}-\mathrm{C}_{6} \mathrm{H}_{4} & \mathrm{n}=1 \\ \text { f } & \mathrm{R}=\mathrm{CH}_{2} \mathrm{CH}\left(\mathrm{CH}_{3}\right)_{2} & \mathrm{Ar}=\mathrm{p}-\mathrm{CH}_{3}-\mathrm{C}_{6} \mathrm{H}_{4} & \mathrm{n}=1\end{array}$


Scheme 2: Proposed fragmentation pattern of compound 3c

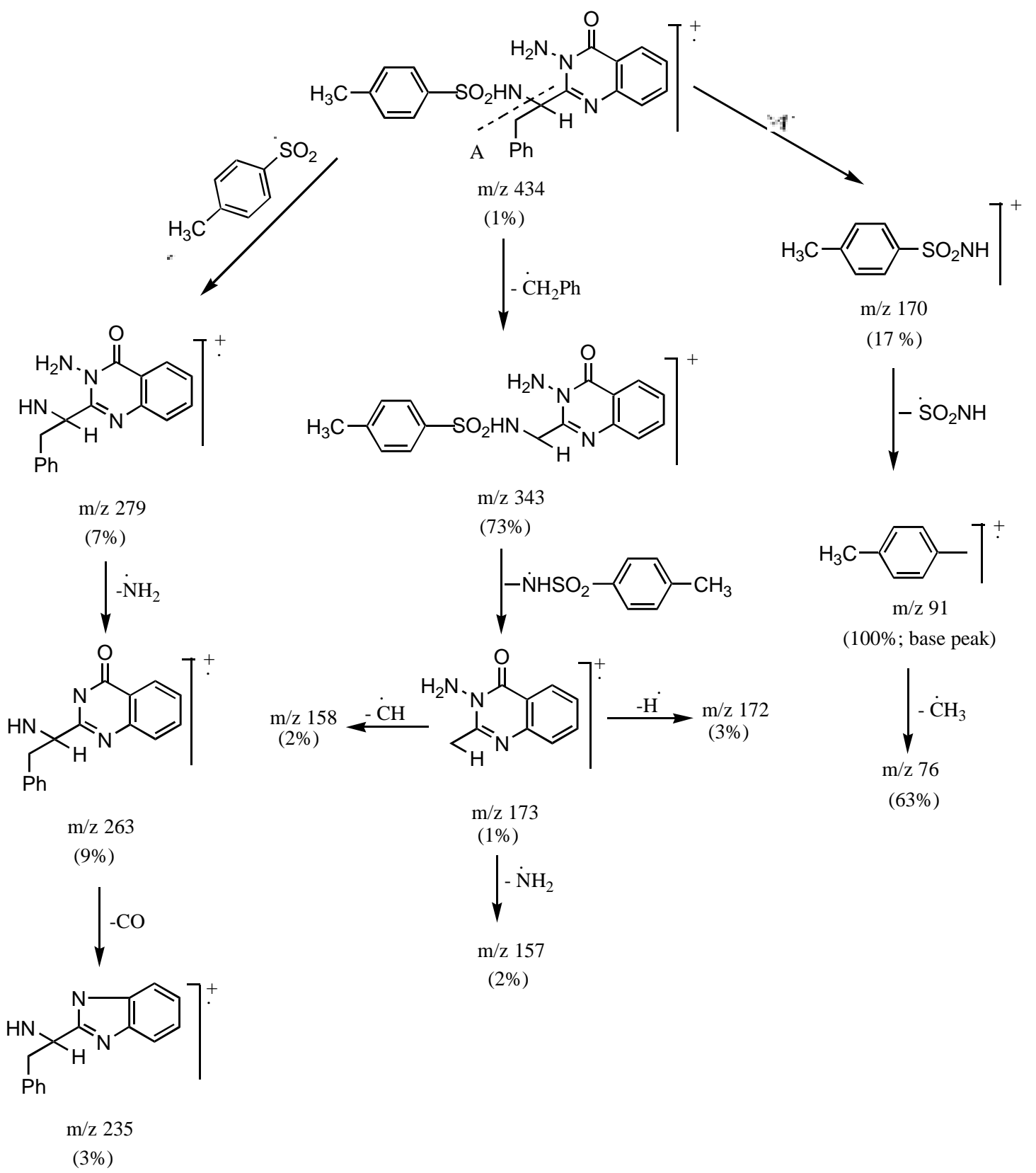

3-Aminoquinazolinone derivatives $\mathbf{3 d , e}$ were used as a precursors for further cyclizations with the purpose of synthesizing several heterocyclic compounds fused to the quinazoline moiety. Thus, condensation of $\mathbf{3 e}$ with p-chlorobenzaldehyde in acetic acid furnished the corresponding Schiff's base 4 . The structure of compound 4 was deduced from elemental analyses and spectral data. The IR spectrum showed the absence of the $\mathrm{NH}_{2}$ group present in the parent compound and the presence of a $\mathrm{C}=\mathrm{N}$ group. On the other hand, refluxing compounds 3d,e with p-fluoro, p-chloro and p-methoxybenzaldehydes in DMF yielded the novel heterocyclic compounds 5a-c. Formation of $\mathbf{5}$ from the reaction of $\mathbf{3}$ and an aldehyde is assumed to proceed in three steps. First, condensation of $\mathbf{3}$ and aldehyde to yield Schiff's bases 4 ; second, nucleophilic addition of $\mathrm{NHSO}_{2}$ to the activated $\mathrm{C}=\mathrm{N}$ bond to form intermediate (A) followed by the third step, elimination of sulphinic acid to furnish $\mathbf{5}$, Scheme 3. 


\section{Scheme 3}<smiles></smiles>

3<smiles>[R]CN(C)C(C)(C)Nn1c(C)nc2ccccc2c1=O</smiles>

A

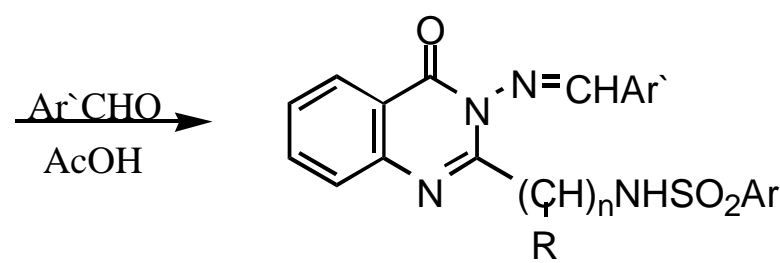

4

DMF

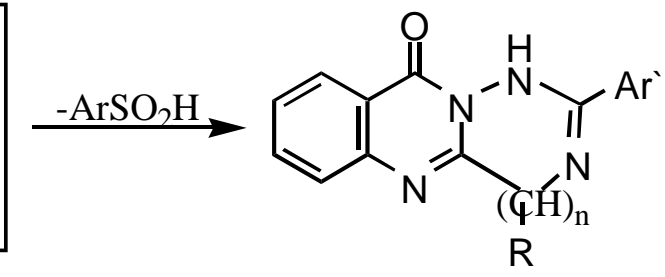

5a-d

\begin{tabular}{|c|c|c|c|}
\hline 4 & $\mathrm{R}=\mathrm{CH}\left(\mathrm{CH}_{3}\right)_{2}$ & $\mathrm{Ar} `=\mathrm{p}-\mathrm{Cl}-\mathrm{C}_{6} \mathrm{H}_{4}$ & $\mathrm{n}=1, \mathrm{Ar}=\mathrm{p}-\mathrm{CH}_{3}-\mathrm{C}_{6} \mathrm{H}_{4}$ \\
\hline $5 \mathbf{a}$ & $\mathrm{R}=\mathrm{H}$ & $\mathrm{Ar}{ }^{\prime}=\mathrm{p}-\mathrm{Cl}-\mathrm{C}_{6} \mathrm{H}_{4}$ & $\mathrm{n}=2$ \\
\hline $5 b$ & $\mathrm{R}=\mathrm{CH}\left(\mathrm{CH}_{3}\right)_{2}$ & $\mathrm{Ar}^{\prime}=\mathrm{p}-\mathrm{F}-\mathrm{C}_{6} \mathrm{H}_{4}$ & $\mathrm{n}=1$ \\
\hline $5 c$ & $\mathrm{R}=\mathrm{CH}\left(\mathrm{CH}_{3}\right)_{2}$ & $A r^{\prime}=p-M e O-C_{6} H_{4}$ & $\mathrm{n}=1$ \\
\hline 5d & $\mathrm{R}=\mathrm{CH}\left(\mathrm{CH}_{3}\right)_{2}$ & $\mathrm{Ar}{ }^{\prime}=\mathrm{p}-\mathrm{Cl}-\mathrm{C}_{6} \mathrm{H}_{4}$ & $\mathrm{n}=1$ \\
\hline
\end{tabular}

Naphtho[2`,3:3,4]-[1,2,5]triazocino[8,1-b]quinazolinone (6a) and naphtho[2`,3 :3,4]-[1,2,5]triazepino[7,1-b]quinazolinone (6b) were obtained by refluxing compounds $\mathbf{3 d}$ and $\mathbf{3 e}$, respectively, with 2,3-dichloro-1,4-naphthoquinone in DMF. This reaction takes place via loss of two $\mathrm{HCl}$ molecules followed by elimination of toluenesulphinic acid.

Also, the 1,2,4-triazepino[7,1-b]quinazolinone 7 and 1,2,4-triazino[6,1-b]quinazolinone 8 were isolated when compounds $\mathbf{3 d}$ and $\mathbf{3 e}$ were refluxed with bis[(methylsulfanyl)methylidene)malononitrile in DMF. The formation of the later compound proceeds via elimination of two methyl mercaptan molecules followed by elimination of toluenesulphinic acid, Scheme 4.

As an extension of this work, when compound 3d was allowed to react with $\mathrm{Cl}\left(\mathrm{CH}_{2}\right)_{\mathrm{n}} \mathrm{COOEt}$ system $(n=0,1)$ in DMF and under reflux conditions, the triazepinoquinazolinone 9 and triazocinoquinazolinone 10 were isolated, respectively. The postulated mechanism for this reaction involves the initial attack by the chloroester followed then by a sequential elimination of ethanol and then toluenesulphinic acid, which is a good leaving group, to give the intermediate (C) which is finally alkylated, Scheme 5 . 
Scheme 4

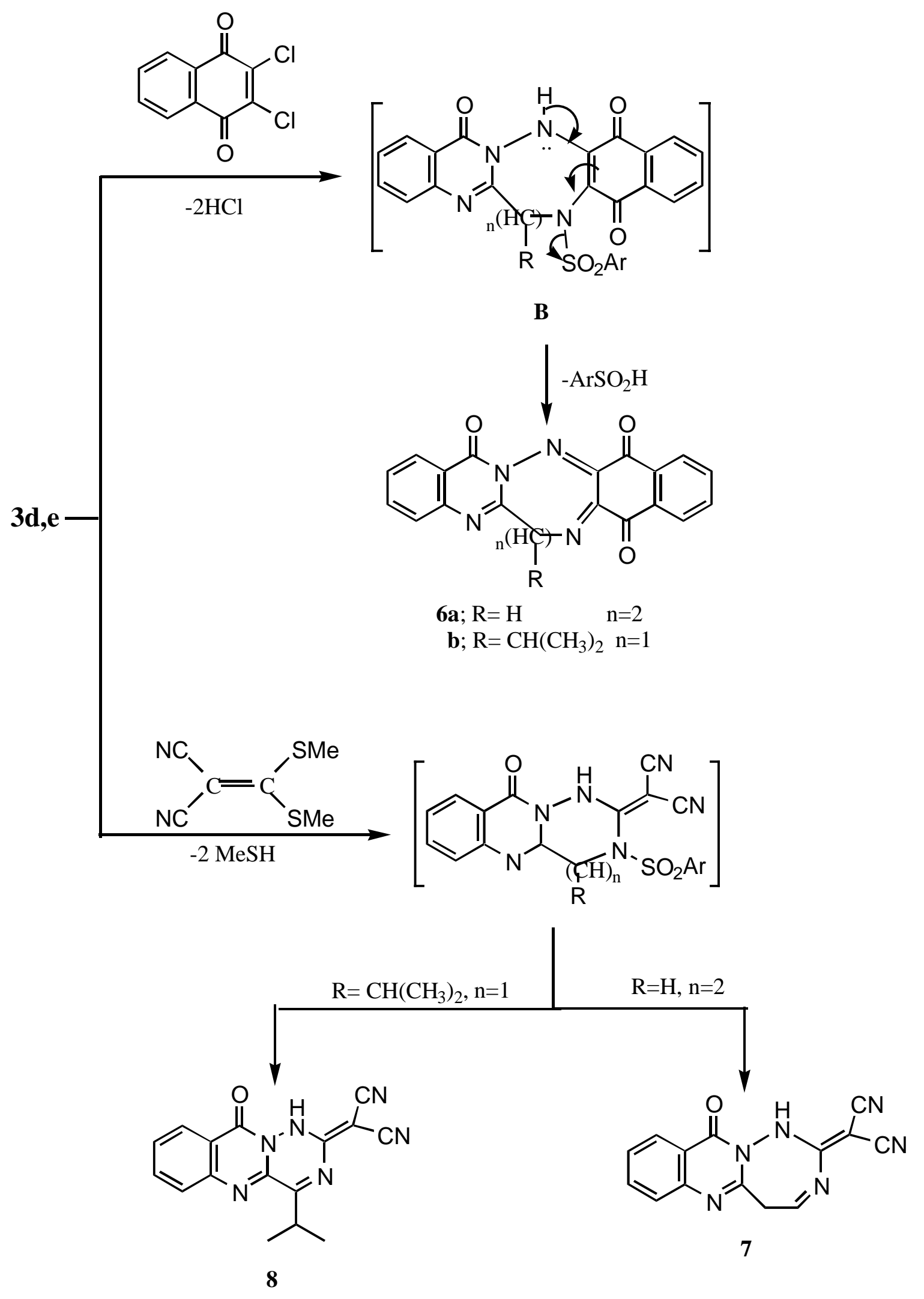




\section{Scheme 5}

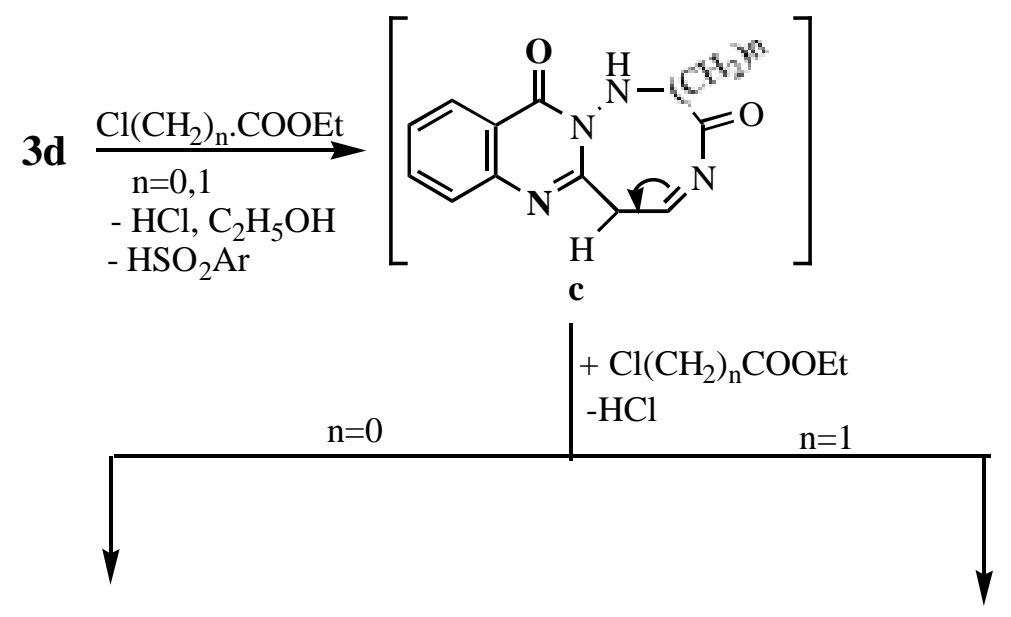<smiles>CCOC(=O)N1C=Cc2nc3ccccc3c(=O)n2NC1=O</smiles>

9<smiles>CCOC(=O)COC1=CNn2c(nc3ccccc3c2=O)CC=N1</smiles>

10

\section{Biological Activity}

\section{Antimicrobial activities}

Compounds $\mathbf{2 b , d , e , ~ 3 a , d , e , f , ~} 9$ and $\mathbf{1 0}$ were tested for their antimicrobial activity by the filter paper disc method [13] using the gram positive bacteria Staphlococcus aureus (NCTC-7447) and Bacillus cerus (ATCC-14579) and the gram negative bacteria Serratia marcesens (IMRU-70) and Proteus merabitis (NCTC-289). The results of antimicrobial activity tests are summarized in Table 1.

Table 1: Antimicrobial screen of the newly synthesized compounds

\begin{tabular}{|c|c|c|c|c|}
\hline \multirow{2}{*}{$\begin{array}{l}\text { Compd. } \\
\text { No. }\end{array}$} & \multicolumn{2}{|c|}{ Gram positive } & \multicolumn{2}{|c|}{ Gram negative } \\
\hline & $\begin{array}{c}\text { Staphlococcus aureus } \\
\text { (NCTC-7447) }\end{array}$ & $\begin{array}{l}\text { Bacillus cereus } \\
\text { (ATCC-14579) }\end{array}$ & $\begin{array}{l}\text { Serratia marcesens } \\
\quad \text { (IMRU-70) }\end{array}$ & $\begin{array}{l}\text { Proteus merabitis } \\
\text { (NTC-289) }\end{array}$ \\
\hline $2 b$ & ++ & ++ & ++ & ++ \\
\hline $2 d$ & + & ++ & ++ & ++ \\
\hline $2 e$ & ++ & ++ & ++ & ++ \\
\hline $\mathbf{3 a}$ & ++ & ++ & ++ & ++ \\
\hline 3d & ++ & +++ & ++ & ++ \\
\hline $3 e$ & +++ & +++ & ++ & +++ \\
\hline $3 f$ & +++ & ++ & +++ & +++ \\
\hline 9 & +++ & ++ & +++ & +++ \\
\hline 10 & ++ & ++ & ++ & ++ \\
\hline
\end{tabular}


Most of the synthesized compounds were found to possess various antimicrobial activities towards all the microorganisms used with minimal inhibitory concentration (MIC). The $\mathrm{N}$-amino compound 3d was found to possess antimicrobial activity against Bacillus cereus (ATCC-14579), compound 3e containing the $\mathrm{N}$-amino moiety possesses a high antimicrobial activity against Staphlococcus aureus (NCTC-7447), Bacillus cereus (ATCC-14579) and Proteus merabitis (NTC289 ) and the $\mathrm{N}$-amino compound $3 \mathbf{f}$ possesses antimicrobial activity towards Staphlococcuss aureus (NCTC-7447), Serratia marcesens (IMRU-70) and Proteus merabitis (NTC-289). Also, compound 9, containing the triazepinoquinazolinone moiety, was found to possess the highest antimicrobial activity towards Staphlococcus aureus (NCTC-7447), Serratia marcesens (IMRU-70) and Proteus merabitis (NTC-289).

\section{Antifungal activities}

The selected compounds were also tested for their antifungal activity using Aspergillus ochraceus Wilhelm (AUCC-230) and Penicillium chrysogenum Thom (AUCC-530) (Table 2). Some of the synthesized compounds were found to possess antifungal activities towards all the microorganisms used with minimal inhibitory concentration (MIC). The amide $\mathbf{2 d}$ possesses a high antifungal activity towards Aspergillus ochraceus Wilhelm (AUCC-230). Also compound 3d which contain the N-amino moiety was found to possess the highest antifungal activity towards Penicillium chrysogenum Thom (AUCC-530). On the other hand the triazepinoquinazolinone 9 was found to possess antifungal activity against Penicillium chrysogenum Thom (AUCC-530).

Table 2: Antifungal activity of the synthesized compounds

\begin{tabular}{|c|c|c|}
\hline Compd.No. & $\begin{array}{c}\text { Aspergillus ochraceus Wilhelm } \\
\text { (AUCC-230) }\end{array}$ & $\begin{array}{c}\text { Penicillium chrysogenum Thom } \\
\text { (AUCC-530) }\end{array}$ \\
\hline $\mathbf{2 b}$ & ++ & ++ \\
$\mathbf{2 d}$ & +++ & ++ \\
$\mathbf{2 e}$ & + & ++ \\
$\mathbf{3 a}$ & $\mathrm{R}$ & $\mathrm{R}$ \\
$\mathbf{3 d}$ & ++ & +++ \\
$\mathbf{3 e}$ & ++ & ++ \\
$\mathbf{3 f}$ & ++ & ++ \\
$\mathbf{9}$ & ++ & +++ \\
$\mathbf{1 0}$ & $\mathrm{R}$ & + \\
\hline
\end{tabular}

Key to the results: Inhibition zones were measured in $\mathrm{mm}$. The concentration used was $4 \times 10^{-5} \mathrm{M}$. Control discs were done using dimethylformamide (DMF) and no inhibition zones were observed. Results are reported as $(\mathrm{R})$ resistant, $(+)$ moderately sensitive giving a $5 \mathrm{~mm}$ inhibition zone; (++) sensitive giving a $14 \mathrm{~mm}$ inhibition zone; (+++) very sensitive giving a $20 \mathrm{~mm}$ inhibition zone. 


\section{Experimental}

\section{General}

Melting points were taken on a STUART apparatus and are uncorrected. The IR spectra $(\mathrm{KBr}$ disks) were measured with a Jasco FT/IR 5300 spectrometer. The ${ }^{1} \mathrm{H}-\mathrm{NMR}$ spectra were obtained on a Varian Gemini 200 instrument at $200 \mathrm{MHz}$ using DMSO- $\mathrm{d}_{6}$ as a solvent and TMS as internal standard. Mass spectra were performed by Shimadzu-GC MS QP 1000 EX using the direct inlet system. Thin layer chromatography (TLC) was carried out on silica gel (MN-Kieselgel G., $0.2 \mathrm{~mm}$ thickness) with ethylacetate:n-hexane (E/H) as the solvent and the plates were scanned under $254 \mathrm{~nm}$ ultraviolet light. Microanalyses were performed by the Microanalytical Unit at Cairo University. All the compounds gave satisfactory elemental analyses. Antimicrobial and antifungal activity tests were carried out by the Microbiology Lab., Faculty of Science, Al-Azhar University, Cairo, Egypt.

General procedure for synthesis of tosyl aminoacids 1a-f.- The tosyl amino acid derivatives were prepared according to McChesney et al [12] whereby the aminoacid (0.026 mol) was dissolved in $1 \mathrm{~N}$ sodium hydroxide $(25 \mathrm{~mL})$ and over a period of thirteen minutes a solution of p-toluenesulphonyl chloride $(0.027 \mathrm{~mol})$ in ether $(30 \mathrm{~mL})$ was added in portions. The mixture was stirred at room temperature for $3 \mathrm{hrs}$. The excess p-toluenesulphonyl chloride was filtered off and the solution treated with $2 \mathrm{~N} \mathrm{HCl}$ until acidic to Congo Red ( $\mathrm{pH} 5$ ). After cooling acidification caused the product to precipitate. The crude product was filtered, washed with water and dried. The crude materials were recrystallized to give $\mathbf{1 a - f}$.

General procedure for synthesis of the amides $2 \boldsymbol{a}$-f. - To a mixture of tosyl aminoacid (0.01 mol) and methyl anthranilate $(0.01 \mathrm{~mol})$ in xylene $(20 \mathrm{~mL})$, phosphorous trichloride $(2-3 \mathrm{~mL})$ was added. The mixture was heated under reflux for 3-4 hrs. After cooling and addition of pet.ether (bp $40-60^{\circ} \mathrm{C} ; 10$ $\mathrm{mL}$ ) the precipitate was filtered and washed with two $5 \mathrm{~mL}$ portions of pet.ether, dried and recrystallized from the appropriate solvent to give 2a-f.

Methyl N-[phenylsulphonyl-glycyl]anthranilate (2a).- From phenylglycyl chloride as colourless crystals (65\%), m.p. $125^{\circ} \mathrm{C}$; IR $v_{\max } / \mathrm{cm}^{-1}$ (2NH), 1703 (ester CO), 1680 (CO).

Methyl N-[4'-methylphenylsulphonyl-2-phenylglycyl]anthranilate (2b).- From tosyl 2-phenylglycyl acid chloride as colourless crystals (71\%), m.p. $155^{\circ} \mathrm{C}$; IR $v_{\max } / \mathrm{cm}^{-1} 3190$ (2NH), 1700 (ester CO), 1681 (CO).

Methyl N-[4'-methylphenylsulphonyl-phenylalaninyl]anthranilate (2c).- From tosyl phenylalaninyl acid chloride as colourless crystals (68\%), m.p. $120^{\circ} \mathrm{C}$; IR $v_{\max } / \mathrm{cm}^{-1} 3195$ (2NH), 1715 (ester CO), 1662 (CO). 
Methyl N-[4'-methylphenylsulphonyl- $\beta$-alaninyl]anthranilate (2d).- From tosyl- $\beta$-alaninyl acid chloride as colourless crystals (73\%), m.p. $130^{\circ} \mathrm{C}$; IR $v_{\max } / \mathrm{cm}^{-1} 3190$ (2NH), 1695 (ester CO), 1660 $(\mathrm{CO})$.

Methyl N-[4'-methylphenylsulphonyl-DL-valinyl]anthranilate (2e).- From tosyl-DL-valinyl acid chloride as colourless crystals $(75 \%)$, m.p. $170^{\circ} \mathrm{C}$; IR $v_{\max } / \mathrm{cm}^{-1} 3220$ (2NH), 1721 (ester CO), 1676 (CO); ${ }^{1} \mathrm{H}-\mathrm{NMR} \delta$ 0.85-0.87 (d, 3H, isopropyl $\mathrm{CH}_{3}$ ), 0.90-0.93 (d, 3H, isopropyl $\mathrm{CH}_{3}$ ), 2.02-2.13 (m, $1 \mathrm{H}, \mathrm{CH}), 2.37$ (d, 1H, CH), $2.45\left(\mathrm{~s}, 3 \mathrm{H}, \mathrm{CH}_{3}\right), 3.98$ (s, 3H, $\left.\mathrm{OCH}_{3}\right), 7.26-8.55$ (m, 8H, ArH's), 11.12 ppm (s, 2H, 2NH).

Methyl N-[4'-methylphenylsulphonyl-DL-leucinyl]anthranilate (2f).- From tosyl-DL-leucine acid chloride as colourless crystals (68\%), m.p. $165^{\circ} \mathrm{C}$; IR $v_{\max } / \mathrm{cm}^{-1} 3180$ (2NH), 1698 (ester CO), 1663 (CO).

General procedure for synthesis of $\mathrm{N}$-amino quinazolin-4(3H)-one derivatives (3a-f).- The corresponding amide 2 a-f $(0.01 \mathrm{~mol})$ and $95 \%$ hydrazine hydrate $(0.05 \mathrm{~mol})$ were dissolved in $\mathrm{n}$ butanol $(30 \mathrm{~mL})$ and refluxed for 6-8 hrs. Cooling in ice gave the crude product which was filtered off and recrystallized to give compounds 3a-f.

3-Amino-2-(phenylsulphonamidomethyl)quinazolin-4(3H)-one (3a).- Colourless crystals (74\%); m.p. $180^{\circ} \mathrm{C}$; IR $v_{\max } / \mathrm{cm}^{-1} 3320\left(\mathrm{NH}_{2}\right), 3210$ (NH), 3060 (CH-arom.), 2960 (CH-aliph.), 1660 (CO).

3-Amino-2-(1 (4-methylphenylsulphonyl)-1 -(phenyl)methyl]quinazolin-4(3H)-one (3b).- Colourless crystals (65\%), m.p. $135^{\circ} \mathrm{C}$; IR $v_{\max } / \mathrm{cm}^{-1} 3325\left(\mathrm{NH}_{2}\right), 3298(\mathrm{NH}), 3060$ (CH-arom.), 2910 (CHaliph.), 1669 (CO), $1598(\mathrm{C}=\mathrm{N})$.

3-Amino 2-[1 (4-methyl phenylsulphonamido)-1 -(benzyl)methyl]quinazo-line-4-(3H)-one (3c).Colourless crystals $(68 \%)$, m.p. $170^{\circ} \mathrm{C}$; IR $v_{\max } / \mathrm{cm}^{-1} 3316\left(\mathrm{NH}_{2}\right), 3241(\mathrm{NH}), 3055$ (CH-arom.), 2972 (CH-aliph.), 1667 (CO); MS $\left(\mathrm{C}_{23} \mathrm{H}_{22} \mathrm{~N}_{4} \mathrm{O}_{3} \mathrm{~S}\right): \mathrm{m} / \mathrm{e} 434\left(\mathrm{M}^{+}, 1 \%\right), 343$ (73.3\%), 328 (10.6\%), 235 (2.7\%), 188 (4.6\%), 172 (2.7\%), 145 (4.2\%), 91 (100\%, base peak), 65 (22.6\%).

3-Amino-2-[4-methyl phenylsulphonamidoethyl]quinazolin-4-(3H)-one (3d).- Colourless crystals (70\%), m.p. $185^{\circ} \mathrm{C}$, IR $v_{\max } / \mathrm{cm}^{-1} 3322,3294\left(\mathrm{NH}_{2}\right), 3260$ (NH), 1666 (CO.), 1614 (C=N).

3-Amino-2-[1 -(4-methylphenylsulphonamido)-1 -(iso-propyl)methyl]quinazolin-4-(3H)-one (3e).Colourless crystals (76\%), m.p. $190^{\circ} \mathrm{C}$; IR $v_{\max } / \mathrm{cm}^{-1} 3320,3290\left(\mathrm{NH}_{2}\right), 3250(\mathrm{NH}), 1665$ (CO), 1611 $(\mathrm{C}=\mathrm{N})$; MS $\left(\mathrm{C}_{19} \mathrm{H}_{22} \mathrm{~N}_{4} \mathrm{O}_{3} \mathrm{~S}\right): \mathrm{m} / \mathrm{e} 387(\mathrm{M}+1,4.5 \%), 353$ (5.6\%), 343 (5\%), 275 (11\%), 226(39.6\%), 185 (15.3\%), 155 (51.6\%), 91 (100\%, base peak), 65 (32\%); ${ }^{1} \mathrm{H}-\mathrm{NMR} \delta$ 0.85-0.92 (d, 6H, 2CH$)$, $1.88\left(\mathrm{~s}, 3 \mathrm{H}, \mathrm{CH}_{3}\right), 2.15-2.24(\mathrm{~m}, 1 \mathrm{H}, \mathrm{CH}), 2.50-2.51(\mathrm{~d}, 1 \mathrm{H}, \mathrm{CH}+\mathrm{DMSO}), 5.51\left(\mathrm{~s}, 2 \mathrm{H}, \mathrm{NH}_{2}\right.$; exchangeable with $\left.\mathrm{D}_{2} \mathrm{O}\right), 6.82-8.02\left(\mathrm{~m}, 8 \mathrm{H}\right.$, Ar-H's), 10.6 (s, $1 \mathrm{H}, \mathrm{NH}$; exchangeable with $\left.\mathrm{D}_{2} \mathrm{O}\right)$. 
3-Amino 2-[1 -(4-methylphenylsulphonamido)-1 -(iso-butyl)methyl]quina-zolin-4-(3H)-one (3f).Colourless crystals (71\%), m.p. $170{ }^{\circ} \mathrm{C}$; ${ }^{1} \mathrm{H}-\mathrm{NMR} \delta$ 0.88-0.94 (d, $\left.6 \mathrm{H}, 2 \mathrm{CH}_{3}\right), 1.52-1.59\left(\mathrm{t}, 2 \mathrm{H}, \mathrm{CH}_{2}\right.$ ), 1.82-1.90 (m, 1H, CH), 2.50-2.52 (d, 1H, CH +DMSO), 5.57 (s, 2H, $\mathrm{NH}_{2}$; exchangeable with $\mathrm{D}_{2} \mathrm{O}$ ), 6.86-8.04 (m, 8H, Ar-H's), 10.2 (s, 1H, NH; exchangeable with $\mathrm{D}_{2} \mathrm{O}$ ).

The Schiff's base of 3-amino-2-[1 -(4-methyl phenyl sulphonamido)-1 -(iso-propyl)methyl]quinazolin-4-(3H)-one (4).- Prepared from 3e (0.01 mol) and 4-chlorobenzaldehyde $(0.01 \mathrm{~mol})$ in acetic acid $(20 \mathrm{~mL})$. The mixture was refluxed for $4 \mathrm{hrs}$., cooled and colected by filteration as pale brown crystals (69\%); m.p. $160^{\circ} \mathrm{C}$; IR $v_{\max } / \mathrm{cm}^{-1} 3220$ (NH), 3050 (CH-arom.), 2985 (CH-aliph.), 1660 (CO).

General procedure for the synthesis of 5a-c.- Compounds $3 \mathrm{~d}, \mathrm{e}(0.01 \mathrm{~mol})$ and the corresponding aldehyde derivative $(0.01 \mathrm{~mol})$ in DMF $(20 \mathrm{~mL})$ containing 2 drops of triethylamine was refluxed for 6-8 hrs., after cooling and acidification with dil. $\mathrm{HCl}$, a precipitate was formed, which was collected by filtration, washed with water and recrystallized to give $\mathbf{5 a - c .}$

2-(4-Chlorophenyl)-4,5,11-trihydro-1H[1,2,4]triazepino[7,1-b]quinazolin-11-one (5a).- Prepared from 3d and 4-chlorobenzaldehyde as brown crystals $(67 \%)$, m.p. $260^{\circ} \mathrm{C}$; IR $v_{\max } / \mathrm{cm}^{-1} 3210(\mathrm{NH})$, $1664(\mathrm{CO}), 1590(\mathrm{C}=\mathrm{N}) ;{ }^{1} \mathrm{H}-\mathrm{NMR} \delta$ 4.23, 4.6 (2s, 4H, 2CH$), 7.2-8.4$ (m, 8H, Ar-H's), 9.2 (s, 1H, $\mathrm{NH}$; exchangeable with $\left.\mathrm{D}_{2} \mathrm{O}\right)$.

2-(4-Fluorophenyl)-4-isopropyl-4,10-dihydro-1H[1,2,4]triazino[6,1-b]-quinazolin-10-one (5b).Prepared from 3e and 4-fluorobenzaldehyde as pale brown crystals $(62 \%)$, m.p. $180^{\circ} \mathrm{C}$; IR $v_{\max } / \mathrm{cm}^{-1}$ 3195 (NH), 2995, 2890 (CH-aliph), 1658 (CO).

2-(4-Methoxyphenyl-4-isopropyl-4,10-dihydro-1H-[1,2,4]triazino[6,1-b] quinazolin-10-one (5c).Prepared from 3e and 4-methoxybenzaldehyde as brown crystals $(60 \%)$, m.p. $170^{\circ} \mathrm{C}$; IR $v_{\max } / \mathrm{cm}^{-1}$ 3210 (NH), 2990, 2885 (CH-aliph.), 1665 (CO).

6,7,9,14,17-Pentahydronaphtho[2`3:3,4][1,2,5]triazocino[8,1-b]qinozolin-9,14,17-trione (6a).Prepared in a similar fashion as 6a from 3d and 2,3-dichloro-1,4-naphthoquinone as deep brown crystals (63\%), m.p. > 300 C; IR v ${ }_{\max } / \mathrm{cm}^{-1} 3060$ (CH-arom.), 1670, 1660 (3CO).

\section{6-Isopropyl-6,8,13,16-tetrahydronaphtho[2‘,3 :3,4][1,2,5]triazepino[7,1-b]-quinazolin-8,13,16}

trione $(\boldsymbol{6} \boldsymbol{b})$.- Prepared from 3e $(0.01 \mathrm{~mol})$; 2,3-dichloro-1,4-naphthoquinone (0.01 mol) in DMF (20 $\mathrm{mL})$ and TEA $(0.05 \mathrm{~mL})$, the reaction mixture was refluxed for $6 \mathrm{hrs}$., cooled and acidified with $\mathrm{HCl}$, the precipitate was filtered, washed with water and recrystallized to give $\mathbf{6 a}$ as dark brown crystals (60\%); m.p. > 300 ${ }^{\circ} \mathrm{C}$; $\mathrm{MS}\left(\mathrm{C}_{22} \mathrm{H}_{16} \mathrm{~N}_{4} \mathrm{O}_{3}\right)$ m/e 384 (5.9\%), 328 (8.1\%), 278 (12.5\%), 224 (14.8\%), 183 (21.7\%), 137 (24.3\%)), 97 (100\%), ${ }^{1} \mathrm{H}-\mathrm{NMR} \delta$ 0.87-0.89 (dd, 3H, isopropyl $\left.\mathrm{CH}_{3}\right), 0.91-0.94(\mathrm{dd}, 3 \mathrm{H}$, isopropyl $\mathrm{CH}_{3}$ ), 2.04-2.15 (m, 1H, isopropyl $\left.\mathrm{CH}\right)$, 7.02-8.34 (8H,m,Ar-H's). 
2-[11-Oxo5,11-dihydro-1H-[1,2,4]triazepino[7,1-b]quinazolin-2-ylidine]-malononitrile (7).- From

3d and [bis(methylthio)methylene]malononitrile as yellow crystals $(61 \%)$; m.p. $250^{\circ} \mathrm{C}$; MS $\left(\mathrm{C}_{14} \mathrm{H}_{8} \mathrm{~N}_{6} \mathrm{O}\right) \mathrm{m} / \mathrm{e} 276$ (3\%), 229 (3.8\%), 203 (26.3\%), 186 (17.6\%), 160 (77.4\%), 91 (100\%, base peak), 65 (46.8\%), ${ }^{1} \mathrm{H}-\mathrm{NMR} \delta 4.3\left(\mathrm{~d}, 2 \mathrm{H}, \mathrm{CH}_{2}\right), 4.5(\mathrm{dd}, 1 \mathrm{H}, \mathrm{CH}), 7.2-8.3(\mathrm{~m}, 4 \mathrm{H}, \mathrm{Ar}-\mathrm{H}$ 's $+1 \mathrm{H}, \mathrm{NH}$; exchangeable with $\mathrm{D}_{2} \mathrm{O}$ ).

\section{2[4-isopropyl-10-oxo-1,10-dihydro-[1,2,4]-triazino-[6,1-b]quinazolin-2-ylidine) malononitrile (8)}

Prepared from 3e $(0.01 \mathrm{~mol})$, [bis(methylthio)methylene)malononitrile $(0.02 \mathrm{~mol})$ in DMF $(20 \mathrm{~mL})$ and TEA $(0.5 \mathrm{~mL})$. The reaction mixture was refluxed for $5 \mathrm{hrs}$. until the odour of methyl mercaptan disappeared. Cooling and acidification caused the precipitation of the product which was then collected and recrystallized to give 7 as pale yellow crystals $(66 \%)$, m.p. $>300^{\circ} \mathrm{C}$; IR $v_{\max } / \mathrm{cm}^{-1} 3210$ $(\mathrm{NH}), 2210(2 \mathrm{CN}), 1659$ (CO).

Ethyl 1-[11-oxo-5,11-dihydro-[1,2,4]triazepino[7,1-b]quinazolinyl]formate (9).- Prepared from 3d $(0.01 \mathrm{~mol})$, ethyl chloroformate $(0.03 \mathrm{~mol})$ and TEA $(0.5 \mathrm{~mL})$ in DMF $(20 \mathrm{~mL})$. The mixture was refluxed for $8 \mathrm{hrs}$., cooled and acidified with dil. $\mathrm{HCl}$. The solid obtained was collected and crystallized to give 9 as pale brown crystals (60\%), m.p. $265^{\circ} \mathrm{C}$; $\mathrm{MS}\left(\mathrm{C}_{14} \mathrm{H}_{12} \mathrm{~N}_{4} \mathrm{O}_{4}\right) \mathrm{m} / \mathrm{e} 300(\mathrm{M}+1$, 0.5\%), 284 (2.9\%), 256 (7\%), 213 (4.9\%), 185 (6.2\%), 149 (16.6\%), 129 (10.8\%), 91 (22.3\%), 65 (8\%); ${ }^{1} \mathrm{H}-\mathrm{NMR} \delta$ 1.28-1.33 (t, 3H, $\left.\mathrm{CH}_{3}\right), 4.23-4.30$ (q, 2H, $\left.\mathrm{CH}_{2}\right), 4.41,4.5(2 \mathrm{~s}, 2 \mathrm{H}, 2 \mathrm{CH}), 7.29-8.29$ (m, 4H, Ar-H's + 1H, NH; exchangeable with $\mathrm{D}_{2} \mathrm{O}$ ).

Ethyl-1-[12-oxo-6,12-dihydro[1,2,5]triazocino[8,1-b]quinazolinyl]acetate (10).- Prepared like 9 from 3d and ethyl chloroacetate as pale brown crystals $(63 \%)$, m.p. > 300 ${ }^{\circ} \mathrm{C}, \mathrm{MS}\left(\mathrm{C}_{16} \mathrm{H}_{16} \mathrm{~N}_{4} \mathrm{O}_{4}\right) \mathrm{m} / \mathrm{e}$ 328 (0.5\%), 299 (0.7\%), 284 (0.8\%), 256 (7.3\%), 213 (4.3\%), 185 (5.2\%), 149 (15.8\%), 69 (100\%; base peak); ${ }^{1} \mathrm{H}-\mathrm{NMR} \delta 1.24-1.32$ (t,3H, $\mathrm{CH}_{3}$-ethyl), $1.80\left(\mathrm{~s}, 2 \mathrm{H}, \mathrm{CH}_{2}\right), 4.09-4.15\left(\mathrm{q}, 2 \mathrm{H}, \mathrm{CH}_{2}\right.$ ethyl), 7.2-8.3 (m, $4 \mathrm{H}$, Ar-H's + 2H, 2NH; exchangeable with $\left.\mathrm{D}_{2} \mathrm{O}\right)$.

\section{References}

1. Daidone, G.; Maggio, B.; Raffa, D.; Plescia, S.; Bajardi, M. L.; Caruso, A.; Cuttuli, V. M. C. and Amico-Roxas, M.: Eur. J. Med. Chem., 1994, 29, 707.

2. A.A.Bckhit and M.A.Khalil, Pharmazie, 1998, 53, 539.

3. Agarwal, R.; Singli, C.and Mishara, N.S.: Ind. Drugs, 1988, 25, 185.

4. Pramella, B.; Rajanarender, E.; Murty, A. K.: Ind. J. Heterocyclic Chem., 1992, 2, 115.

5. Shyimad, M.; Kalsi, R.; Dixit, K. S.and Barthwal, J. P.: Arzneim-Fosch. Drug Res., 1991, 41, 514.

6. Shakhidoyator, Kh. M.; Eripor, E. O.; Yun, L. M.; Yamankulov, M. Ya and Kadyrov, Ch. Sh.; Fungitsidy, 1980, 66; Chem. Abstr.: 1981, 94, 192253.

7. Fetter, J.; Czuppon, T.; Hornyak, G. and Feller, A.: Tetrahedron, 1991, 47, 9393.

8. Kralj, L.; Hvala, A.; Sreto, J.; Golic, L. and Stanovik, B.: J. Heterocyclic Chem., 1997, 34, 247. 
9. Mohamed, Y. A.; El-Sharief, A. M. Sh.; Ammar, Y. A.; Amin, N. E. and Ghorab, M. M.: J. Serb. Chem. Soc., 1989, 54, 179.

10. Ammar, Y. A.; El-Sharief, A. M. Sh.; Mohamed, Y. A. and Ahmed, H. A.: J. Serb. Chem. Soc., 1987, 52, 633.

11. Hassanein, A. A.; Nassar, O. M.; Zahran, M. A. and Ali, A. H.: Al-Azhar Bull. Sci., 1997, 8, 417.

12. McChesney, E.W. and Kirkswann, W. M.: J. Am. Chem. Soc., 1937, 59, 1116.

13. Elslager, E.F.: Prog. Drug. Res., 1974, 18, 99.

Sample Availability: Samples are available from the authors.

(C) 2000 by MDPI (http://www.mdpi.org). Reproduction is permitted for noncommercial purposes 